\title{
Intelligent Telemetric Implants
}

\author{
M. Schuettler ${ }^{1,2}$ and T. Stieglitz ${ }^{1,2,3}$ \\ ${ }^{1}$ Lab. for Biomedical Microtechnology, Dept. for Microsystems Engineering, Freiburg, Germany, schuettler@ieee.org \\ ${ }^{2}$ CorTec GmbH, Freiburg, Germany \\ ${ }^{2}$ Bernstein Center Freiburg, Germany
}

\begin{abstract}
All today's active implantable medical devices utilize wireless telemetry, serving at least one of the two purposes: Power transmission and/or communication with an extra corporal unit. For implant powering, three strategies are widely used: 1. Primary (non-rechargeable) batteries, that require the presence of a system providing information on battery condition, warning the user/practitioner of an upcoming battery exhaustion. Usually, the implementation of such a system employs a telemetric data link. 2. Inductive coupling, using an extra corporal transmitter coil generating an alternating magnetic field, which couples through the skin into an implanted receiver coil that converts the magnetic flux into an electrical supply voltage. 3. Secondary batteries that are periodically recharged using an inductive link. Here, a power monitoring system is mandatory, commonly implemented by a telemetric link. Besides the telemetric links associated with power supply and its monitoring, implants communicate with extra-corporal units in order to transmit recorded physiological data, to report implant malfunction or critical conditions, to be re-programmed (e.g. adaptation of stimulation intensity in pacemakers), or in future applications, to communicate with other implants within a bodyinternal wireless network.
\end{abstract}

\section{Introduction}

The first implanted cardiac pacemaker was a stand-alone battery-operated device that delivered electrical stimuli to the heart at constant repetition rate. Today, 55 years later, pacemakers are highly complex systems, that record and analyze heart activity, measure electrode impedance, store physiological as well as system status data, react on biological demand and communicate with extra corporal units (ECUs) without the patient's notice. Communication takes place via radio frequency, as many implanted devices do today. The international standard EN 40552-1 demands that implants with energy storage to incorporate a system for reporting depletion of energy. In praxis, such a system is implemented as a telemetric link. In the following sections, we briefly review concepts of modern implant telemetry.

\section{Telemetric Powering}

Powering of implanted devices by a telemetric link is widely used across many applications. It can either be applied for periodic recharging of secondary cells or for direct powering of implants without an energy storage. The vast majority of implant systems utilize inductive coupling between an ECU and the implanted part. In general, this link can be described as a lossy transformer. An alternating current flows through the primary coil of the ECU, producing an alternating magnetic field. Parts of this field permeate the secondary coil of the implant, inducing an alternating voltage. After rectifying and smoothing, the voltage is regulated to a fixed value, supplying the electronic implant circuitry. Besides other influences, the efficiency of such an inductive link strongly depends on the distance between the transmitting and the receiving coil and the coil diameters. In general the coil diameter $d$ for a given maximum distance $x$ should be $d \geq 2 \cdot x \cdot 2^{1 / 2}=2.83 \cdot x[1]$.

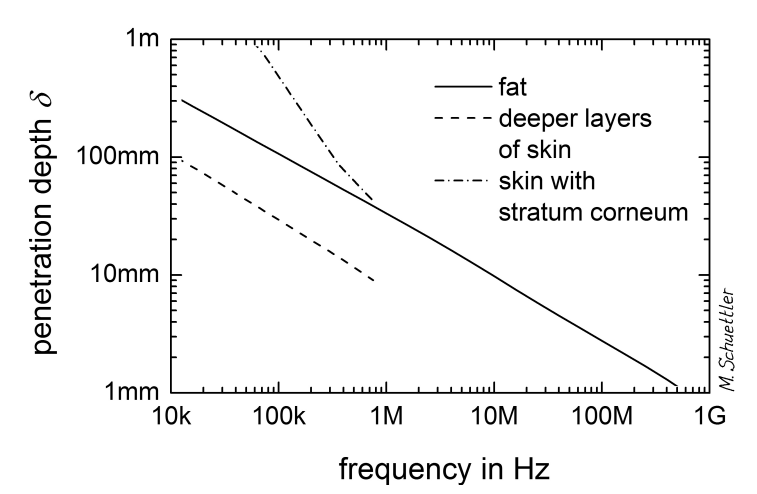

Figure 1: Different layers of biological tissue (skin thickness: a couple of $10 \mu \mathrm{m}$ to a few $\mathrm{mm}$, fat: up to a few $10 \mathrm{~mm}$ ) have different frequency-dependent electrical conductivities and hence the magnetic field penetrates into different depths. In general, magnetic fields at low frequencies pass tissue with less losses than high frequency fields. Data was extracted from [2] and [3].

The choice of magnetic field frequency $f$ is affected by aspects as frequency-dependent attenuation by eddy currents induced in the biological tissue between the implant and the ECU. A measure for describing this effect is the penetration depth $\delta=\left(\mu_{0} \mu_{r} \sigma \pi \cdot f\right)^{-1 / 2}$ at which the field is reduced in its strength by a factor of $1 / e \approx 0.368$ (see Figure 1), with $\mu_{0}=4 \pi \cdot 10^{6} \mathrm{VA} / \mathrm{ms}, \mu_{r} \approx 1$ (tissue), $\sigma=$ electrical conductivity. If the receiver coil is placed inside a hermetic package, the field strength is further reduced, strongly dependent on the electrical conductivity and thickness of the package shell (Figure 2). The attenuation of the magnetic field by tissue and hermetic metal packages becomes stronger with in creasing frequencies, suggesting the use of low field freqeuencies. Furthermore, power amplifier circuits needed for generating strong primary coil currents become less efficient at high frequencies beyond some MHz [4]. Last but not least, legislation restricts the use of the frequency spectrum, assigning a radiated power maximum to each frequency band. 


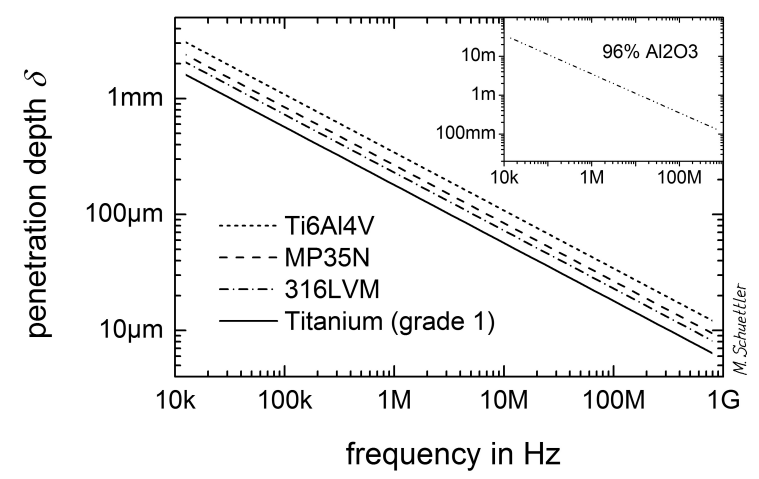

Figure 2: Penetration depth in hermetic implant shells decreases with increasing frequency and with increasing electrical conductivity of the shell material. A common metal shell thickness is 127 to $250 \mu \mathrm{m}$. Data on permeability and conductivity was extracted from biomaterialienkatalog.de, azom.com, matweb.com, ceramtec.com (accessed: 1 July 2012).

Besides some national exceptions, the use of the radio frequency spectrum for short range devices (SRD) in Europe is regulated by the recommendation ERC/RE 7003 of the European Radiocommunications Committee (ERC). Annex 12 assigns frequency bands to be used by active medical implants and their associated peripherals. Table 1, lists the maximum radiated power / magnetic field strength for the individual bands. ERC/RE 70-03 introduces further restrictions, such as band width, channels spacing, restrictions to indoor use and/or animal implantations and refers to the harmonized international standards (EN $300 \mathrm{xxx}$ to EN 0302 yyy) defining technical specifications of the individual bands.

Table 1: Frequency spectrum for short range active medical implants and associated peripherals (e.r.p. $=$ effective radiated power, e.i.r.p. $=$ effective isotopic radiated power)

\begin{tabular}{|c|c|c|c|}
\hline Band & Freq. Range & Power/ Magn.Field & Duty Cycle \\
\hline a & $402-405 \mathrm{MHz}$ & $25 \mu \mathrm{W}$ e.r.p. & n.a. $*$ \\
\hline a1 & $401-402 \mathrm{MHz}$ & $25 \mu \mathrm{W}$ e.r.p. & $\leq 0.1 \% *$ \\
\hline a2 & $405-406 \mathrm{MHz}$ & $25 \mu \mathrm{W}$ e.r.p. & $\leq 0.1 \% *$ \\
\hline b & $9-315 \mathrm{kHz}$ & $30 \mathrm{~dB} \mu \mathrm{A} / \mathrm{m}$ at $10 \mathrm{~m}$ & $<10 \%$ \\
\hline c & $315-600 \mathrm{kHz}$ & $-5 \mathrm{~dB} \mu \mathrm{A} / \mathrm{m}$ at $10 \mathrm{~m}$ & $<10 \%$ \\
\hline $\mathrm{d}$ & $30-37.5 \mathrm{MHz}$ & $1 \mathrm{~mW}$ e.r.p. & $<10 \%$ \\
\hline e & $12.5-20 \mathrm{MHz}$ & $-7 \mathrm{~dB} \mu \mathrm{A} / \mathrm{m}$ at $10 \mathrm{~m}$ & $<10 \%$ \\
\hline f & $2.4835-2.5 \mathrm{GHz}$ & $10 \mathrm{dBm}$ e.i.r.p & $<10 \% *$ \\
\hline
\end{tabular}

Implants are also allowed to operate using the Industry, Science and Medical (ISM) bands for SRDs, regulated by the International Telecommunication Union (ITU). However, ISM bands are less attractive to be used by implants because of the comparably large interference originating from ISM devices of everyday use, e.g. radio identification (RFID) tags, remote controlled models, microwave ovens, anti-theft devices, Bluetooth, wireless local area networks (WLAN), ZigBee, etc. Table 2 list potential ISM bands for implant use. Across individual nations, ISM bands might slightly differ or even have additional bands.
Table 2: Harmonized frequency spectrum for the ISM band. Type A: Operation requires certification. Type B: No certification required, strong interference expected [1]

\begin{tabular}{|c|c|c|}
\hline Type & Freq. Range & $\begin{array}{c}\text { Power/ } \\
\text { Magn.Field }\end{array}$ \\
\hline A & $6.765-6.795 \mathrm{MHz}$ & $42 \mathrm{~dB} \mu \mathrm{A} / \mathrm{m}$ at $10 \mathrm{~m}$ \\
\hline B & $13.553-13.567 \mathrm{MHz}$ & $42 \mathrm{~dB} \mu \mathrm{A} / \mathrm{m}$ at $10 \mathrm{~m}$ \\
\hline B & $26.957-27.283 \mathrm{MHz}$ & $42 \mathrm{~dB} \mu \mathrm{A} / \mathrm{m}$ at $10 \mathrm{~m}$ \\
\hline B & $40.66-40.7 \mathrm{MHz}$ & $10 \mathrm{~mW}$ e.r.p. \\
\hline A & $433.05-434.79 \mathrm{MHz}$ & $10 \mathrm{~mW}$ e.r.p. ${ }^{*}$ \\
\hline B & $2.4-2.483 \mathrm{GHz}$ & $10 \mathrm{~mW}$ e.i.r.p. \\
\hline B & $5.725-5.875 \mathrm{GHz}$ & $25 \mathrm{~mW}$ e.i.r.p. \\
\hline & \multicolumn{2}{|c|}{ (continued in GHz range) } \\
\hline
\end{tabular}

* depends on duty cycle

Implanted devices utilizing inductive powering are usually non-commercial, investigational devices, e.g. implantable brain-computer-interfaces [5] or retinal implants [6]. Commercial implants use inductive powering in combination with magnetic field modulation, allowing for data transfer via the same link. This type of implant telemetry is described in the following section.

\section{Telemetric Powering and Communication with an ECU}

A simple method of combining powering and communication using the same pair of coils is coding information in the amplitude of the alternating magnetic field. The amplitude changes are detected by the implant and the information is decoded. This so-called amplitude modulation (AM) has a digital variation, in which the carrier (the magnetic field) is switched between two amplitudes, representing a logic 'low' or 'high' state. While 'high' might be a full carrier amplitude, 'low' is represented by an amplitude between 0 (magnetic field is switched off) or some fraction of the full carrier amplitude. The so-called modulation depth describes to which fraction the carrier amplitude is modulated. $100 \%$ modulation depth means: switching the carrier off. This digital modulation technique is referred to as amplitude shift keying (ASK) and is very popular in short range implant communication. Other modulation strategies utilise modulation of the frequency of the carrier (FM) or switching between two carrier frequencies: frequency shift keying (FSK). A third technique changes the phase of the carrier by $180^{\circ}$, or fractions of it, named phase shift keying (PSK). In praxis, the aforementioned modulation techniques usually permit a data rate of up to $10 \%$ of the carrier frequency. More advanced modulation techniques allow higher data rates, e.g. combining PSK and ASK. In general, the maximum data rate is proportional to the carrier frequency. This is the main reason, why not all commercial implants utilize low frequencies as suggested in the previous section in order to minimize electromagnetic losses. In fact, the choice of the carrier frequency is dominated by the data rate required to operate the implant. Neuromodulators, 
e.g. for treatment of neuropathic pain, require only a very low data rate for re-programming and for exchange of status information such as battery charge level, electrode impedance, etc. This type of implant, however, requires a minimum loss magnetic link in order to minimize charging times and tissue heating. Hence the lower frequency spectrum from $43.4 \mathrm{kHz}$ (Boston Scientific) up $175 \mathrm{kHz}$ (Medtronic) is used [7], allowing data rates of up to $12.5 \mathrm{kBd}$ [8]. In contrast, cochlear implants use a constant stream of data at high rate, providing hearing sensation to profound deaf persons. Here, carrier frequencies between $5 \mathrm{MHz}$ (Cochlear Ltd.) and $49 \mathrm{MHz}$ (Advanced Bionics Corp.) are used, so data can be transferred at a rate up to $1.09 \mathrm{Mbit} / \mathrm{s}$ [9].

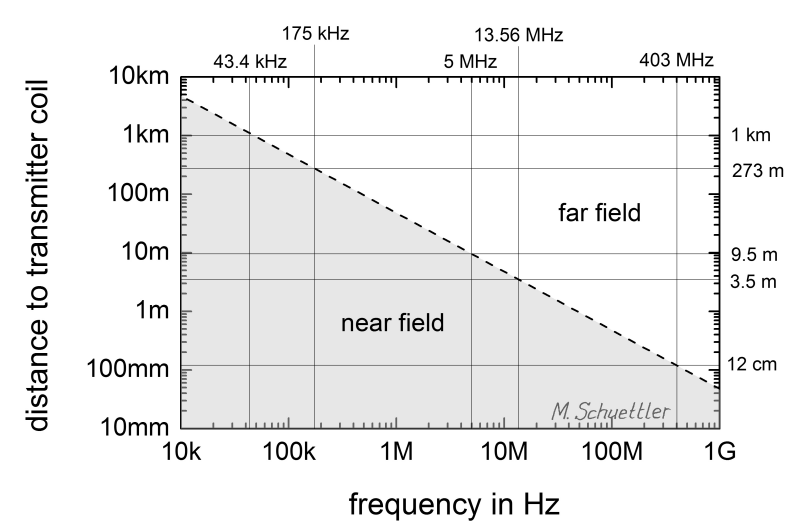

Figure 3: Transition from near to far field as function of carrier frequency. Note: The transistion is of gradual nature and small variations of the definition of the transition exist in literature.

All these implants do not only receive data from the ECU but they respond, too. As long as the implant is in close proximity to the primary coil, a transformer-like situation exists, in which the electrical impedance of the implant electronic circuit is reflected back to the transmitter circuit. This means that the actual transmitted power depends on the electrical impedance of the implant. By modulating the implant impedance, the transmitted power is modulated, which - in turn - can be detected by the transmitter. In praxis, the impedance of the implant is modulated by an electronic switch, which switches between two impedance states. Since the implant is seen by the ECU as an electrical load, this very simple and commonly applied method is called load shift keying (LSK). The maximum achievable LSK data rate is proportional to the carrier frequency. As mentioned before, LSK depends on the modulation of the magnetic field generated by the ECU. However, depending on the carrier frequency, the magnetic field generated by a primary coil gradually transforms into an electric field with increasing distance to the coil. The electric field however, can not be modulated by LSK. The transition from the (electro-)magnetic to the electric field takes place at about $d_{L}=c /(2 \pi \cdot f)$ with $c$ being the speed of light and $f$ the frequency of the field. At a distance of $d<d_{L}$, the field is called near field, while the so-called far field begins to dominate at larger distances. The field strength of the near field decreases proportional to $1 / d^{3}$ while the far field decreases with $1 / d$. Figure 3 shows that LSK cannot be used for frequencies above $1 \mathrm{GHz}$, considering implantation depth of a few centimetres. [1]

\section{Communication with an ECU without Telemetric Powering}

In case an implant is not inductively powered, but rather uses primary cells, as a cardiac pacemaker, the communication can be shifted to the far field. Since the implant does not harvest energy from the transmitted field but only decodes information, the transmission power can be very small. Outside the body, the attenuation of the far field is low and therefore, the ECU does not have to be placed in close proximity to the implant. Far field communication is applied in modern cardiac pacemakers that communicate with an ECU (in this context also called: 'wand') over a distance of $2 \mathrm{~m}$ or more. In praxis, this is used e.g. on a daily basis to transfer status information recorded and stored in the pacemaker to the wand, which is usually placed next to the bed connected to the telephone landline (Medtronics, Boston Scientific, St. Jude Medical) or is carried as a portable device on the belt, linked to the GSM mobile phone network (Biotronik). The wand relays the data to a server accessible by the medical peer. In case of a critical event reported by the pacemaker, the patient has to see a physician. By this way, periodical health checks of the patient can be dramatically reduced in number without compromising the health care quality. This type of telecare is state of the art for all major pacemaker suppliers. The frequencies band used is named Medical Implant Communication Service (MICS, 402-405 MHz) [10]. An additional advantage of far field communication is the functional testing and programming sequence of the implant during initial surgery. Using an ECU employing near field communication is considered a hazard to the sterility of the wound, while a far field ECU is used up to $2 \mathrm{~m}$ away from the patient.

\section{Communication with other Implants: Body Area Networks}

Future development of implanted technology might involve a communication network of different implants and ECUs. Currently, standards for body area networks (BAN) are developed to insure safe and secure data exchange in a range of $2 \mathrm{~m}$. The physical layer including carrier frequencies, transmission power, protocols, bandwidth etc. undergoes standardisation by a task group (IEEE 802.15.BAN) that previously defined the popular Bluetooth standard. BAN are divided into two areas of application: 1. Networks on the body: 'Wearable BAN' and 2. Networks inside the body: 'Implant BAN'. Also the standard is not passed yet, the operational band will most likely be $2.36 \mathrm{GHz}$ to $2.4 \mathrm{GHz}$. A data transmission rate of $10 \mathrm{Mbit} / \mathrm{s}$ is targeted and a strong emphasis is put on data security, physical patient safety (e.g. specific absorption rate, SAR), low power, small implementation size and very high reliability [11], [12]. Examples for potential Implant-BANs are pacemakers that communicate with implanted nerve activity monitors or implanted insulin 
drug pumps communicating with glucose sensors. [11] Currently, implant-implant communication systems are under development. The Alfred Mann Foundation, e.g., works on a new generation of their injectable microstimulator $B I O N$. While one type of these units is capable of delivering electrical stimuli, other units sense acceleration, bioelectric signals, tissue displacement, and position/orientation [13]. Connecting multiple of these units aims at forming a wireless artificial nervous system, providing a better control over artificial muscle/organ activation and as a result, more 'natural' locomotion or other forms of neuro-muscular therapy. Data is exchanged within the body in a frequency band of $413-457 \mathrm{MHz}$ (Medical Micro-Power Networks), allocated especially for this application by the US American authorities, the Federal Communication Commission in early 2012 [14].

\section{Alternative Concepts}

Although near and far field radio frequency communication dominates implant technology, other means of telemetric powering and data transfer have been investigated. Optical data transmission becomes attractive when high data rates have to be transmitted [5], [6]. Besides optical powering of implants has also been shown to be generally feasible [15]. The drawback of magnetic field attenuation by biological tissue encourage research groups to investigate ultrasonic powering of implants. It was shown that ultrasonic power and data transfer becomes more efficient than radio frequency once the implants are placed in deep areas of the body. Even an analogy to LSK is possible by changing the implant's acoustic impedance, resulting in a different echo, detectable by the ECU [16]. Furthermore, direct implant powering using capacitive coupling was investigated [17]. However, none of these methods is currently applied in commercial implants.

\section{Conclusion}

Implant telemetry is a very important, dynamically developing and also highly regulated field, addressing aspects as remote power supply and communication of implants and extra corporal units. Newer developments include implanted body networks and implants communication linked to mobile phone networks.

\section{References}

[1] Finkenzeller K: RFID Handbuch. Muenchen: Carl Hanser Verlag, 4. Auflage, 2006

[2] Miklavcic D, Pavselj N, Hart FX: Electrical Properties of Tissues. Wiley Encyclopedia of Biomedical Engineering, pp. 1-12, 2006

[3] Andreuccetti D: Dielectric Properties of Body Tissues. http://niremf.ifac.cnr.it/tissprop/, accessed: 30 June 2012

[4] Troyk PR, Brown IE, Moore WH, Loeb GE: Development of BIONTM Technology for Functional Electrical Stimulation: Bidirectional Telemetry. Proc. IEEE EMBC, Vol 2, pp. 1317-1320, 2001
[5] Schuettler M, Kohler F, Ordonez JS, Stieglitz T: Hermetic Electronic Packaging of an Implantable Brain-Machine-Interface with Transcutaneous Optical Data Communication, Proc IEEE EMBC, in press, 2012.

[6] Ordonez JS, Schuettler M, Ortmanns M, Stieglitz T: A 232-Channel Retinal Implant with a Miniaturized Hermetic Package, Proc IEEE EMBC, in press, 2012.

[7] Shah AJ, Brunett JD, Thaker JP, Patel MB, Liepa VV, Jongnarangsin K, Thakur RK: Characteristics of Telemetry Interference with Pacemakers Caused by Digital Media Players. Pacing Clin Electrophysiol, Vol 33(6), pp. 712-720, 2010

[8] Halperin D, Heydt-Benjamin TS, Ransford B, Clark SS, Defend B, Morgan W, Fu K, Kohno T, Maisel WH: Pacemakers and Implantable Cardiac Defibrillators: Software Radio Attacks and Zero-Power Defenses. Proceedings of IEEE Symp. on Security and Privacy, pp. 129-142, 2008

[9] Zeng F-G, Rebscher S, Harrison WV, Sun X, Feng $\mathrm{H}$ : Cochlear Implants: System Design, Integration and Evaluation. IEEE Rev Biomed Eng, Vol 1, pp. 115-142, 2008

[10] Lakshmanadoss U, Shah A, Daubert JP: Telemonitoring of the Pacemaker. In: Modern Pacemakers Present and Future. Ed: Das MK, Rijeka: InTech, 2011.

[11] Li H-B, Kohno R: Body Area Network and Its Standardization at IEEE 082.15.BAN. Advances in Mobile and Wireless Communications, Lecture Notes in Electrical Engineering, Vol. 16 (IV), pp. 223-238, DOI: 10.1007/978-3-540-79041-9_12, 2008

[12] The Institute of Electrical and Electronics Engineers: IEEE 802.15 WPANTM Task Group 4j (TG4j) Medical Body Area Networks. http://www.ieee802.org/ 15/pub/TG4j.html, accessed: 9 July 2012

[13] Loeb GE, Richmond FJR, Singh J, Peck RA, Tan W, Zou Q, Sachs N: RF-Powered BIONs ${ }^{\mathrm{TM}}$ for Stimulation and Sensing. Proceedings of IEEE EMBC, Vol 2, pp. 4182-4185, 2004

[14] Federal Communication Commission (FCC): Small Entity Cimpliance Guide - Medical Micro-Power Networks. http://transition.fcc.gov/Daily_Releases/ Daily_Business/2012/db0509/DA-12-737Ā1.pdf accessed: 9 July 2012

[15] Goto K, Nakagawa T, Nakamura O, Kawata S: An Implantable Power Supply with an Optically Rechargeable Lithium Battery. IEEE Trans Biomed Eng, Vol 48(7), pp. 830-833, 2001

[16] Arra S, Leskinen J, Heikkila J, Vanhala J: Ultrasonic Power and Data Link for Wireless Implantable Applications. Proceedings of the IEEE Symp. on Wireless Pervasive Computing, pp. 567-571, 2007

[17] Sodagar AM, Amiri P: Capacitive Coupling for Power and Data Telemetry to Implantable Biomedical Microsystems. Proceedings of the IEEE NER, pp. 11-414, 2009 